\title{
Comparison of Early Evolutions of Mimas and Enceladus
}

\section{Leszek CZECHOWSKI and Piotr WITEK}

Institute of Geophysics, University of Warsaw, Warszawa, Poland

e-mail: lczecho@op.pl (corresponding author)

\begin{abstract}
Thermal history of Mimas and Enceladus is investigated from the beginning of accretion to $400 \mathrm{Myr}$. The numerical model of convection combined with the parameterized theory is used. The following heat sources are included: short lived and long lived radioactive isotopes, accretion, serpentinization, and phase changes. The heat transfer processes are: conduction, solid state convection, and liquid state convection. We find that temperature of Mimas' interior was significantly lower than that of Enceladus. If Mimas accreted 1.8 Myr after CAI then the internal melting and differentiation did not occur at all. Comparison of thermal models of Mimas and Enceladus indicates that conditions favorable for the start of tidal heating lasted for a short time $\left(\sim 10^{7} \mathrm{yr}\right)$ in Mimas and for $\sim 10^{8} \mathrm{yr}$ in Enceladus. This could explain the Mimas-Enceladus paradox. In fact, in view of the chronology based on cometary impact rate, one cannot discard a possibility that also Mimas was for some time active and it has the interior differentiated on porous core and icy mantle.
\end{abstract}

Key words: medium-sized satellites, thermal evolution, differentiation, Mimas-Enceladus paradox.

\section{INTRODUCTION}

Enceladus and Mimas are two smallest medium-sized satellites (MIS) of Saturn. The group consists of 6 spherical satellites: Mimas, Enceladus, Tethys, Dione, Rhea, and Iapetus. They are built of mixtures of rocks and

Ownership: Institute of Geophysics, Polish Academy of Sciences;

(c) 2015 Czechowski and Witek. This is an open access article distributed under the Creative Commons Attribution-NonCommercial-NoDerivs license,

http://creativecommons.org/licenses/by-nc-nd/3.0/. 
ices. The rocky component is believed to be of chondritic composition. The main component of ices is frozen $\mathrm{H}_{2} \mathrm{O}$ but some admixture of ammonia and other volatiles is expected (e.g., Peale 2003).

Since the flyby of the Voyager probe it is known that Mimas has a heavily cratered surface devoid of features that would indicate recent endogenic activity. The majority of craters are generally thought to form during the Late Heavy Bombardment Epoch. Lineaments visible on the surface of this satellite are thought to be a result of the impact that created the Herschel crater, although freezing expansion cannot be ruled out a priori (Jaumann et al. 2009). The density of Mimas $\left(1147 \mathrm{~kg} \mathrm{~m}^{-3}\right)$ is much lower than that of Enceladus $\left(1609 \mathrm{~kg} \mathrm{~m}^{-3}\right)$. This indicates that ices constitute important part of this body and the rock content is lower in Mimas than in Enceladus, which in turn suggests lower heating rate due to radiogenic isotopes. The shape of Mimas determined by the Cassini probe might suggest internal mass concentration (due to differentiation and formation of the core). The small deviation from the hydrostatic ellipsoid shape limits what can be inferred about the interior (Thomas 2010). The significant radial porosity gradient, as hypothesized by Eluszkiewicz (1990), is now thought to be short-lived (LeliwaKopystyński and Kossacki 2000).

Mimas has a relatively large eccentricity $(e=0.02)$ which is thought to be primordial (e.g., Matson et al. 2009); however, it could also be explained by the passage of the satellite through mean motion resonances with either Enceladus or Dione (Meyer and Wisdom 2008). To sustain the primordial eccentricity, the Mimas' interior must have been cold and rigid through most of its history (McKinnon and Barr 2007). Calculations of Multhaup and Spohn (2007) indicate that partial melting and differentiation of Mimas is possible only if some ammonia is present and the nebula ambient temperature is around $250 \mathrm{~K}$. However, they did not consider the heat of short-lived radioactive isotopes, like ${ }^{26} \mathrm{Al}$. Moreover, Muro and Nimmo (2011) calculated that initial temperatures exceeding $220 \mathrm{~K}$ lead to runaway dissipation scenario and fast eccentricity damping. Charnoz et al. (2009) suggested that during the Late Heavy Bombardment (LHB) Mimas could have experienced catastrophic disruption and reassembled thereafter; in contrast, survival of Enceladus is much more probable in each considered model.

Data concerning interiors of the MIS are very scarce. The most direct data are measurements of gravitational field and magnetic field of the satellites. From these data one can obtain mass distribution and distribution of electric conductivity (high conductivity means a probable existence of liquid water); see, e.g., Bobojć and Drożyner (2011), Essa (2007), Kriegel et al. (2009). However, even these data are rather limited. The best situation is for Enceladus because of several close flybys and determination of chemistry of the plumes over the South Polar Terrain (SPT). 
A few papers considered early thermal evolution of Enceladus. First models, like Ellsworth and Schubert's (1983), do not include short-lived isotopes (SLI). They found that the heating from long-lived radioactive isotopes (LLI) was not intensive enough for melting the interior but it was large enough to support solid state convection for 3.3 billion years. This convection could prevent extensive melting.

Schubert et al. (2007) investigated first $20 \mathrm{Myr}$ of Enceladus' history. They have found that the interior of Enceladus experienced early differentiation due to melting of ice from radiogenic heating (mainly ${ }^{26} \mathrm{Al}$ ). At 10 to $20 \mathrm{Myr}$ after formation, Enceladus had hot rocky core of $165 \mathrm{~km}$ size. However, this conclusion depends on time of accretion. If Enceladus accreted 3.5 Myr after the formation of Calcium-aluminum-rich inclusions (CAI), its interior would not melt, unless the initial temperature was high. If the temperature was $200 \mathrm{~K}$ at the time of Enceladus' formation, then differentiation occurred even for low content of ${ }^{26} \mathrm{Al}$, but it would not be complete. Their model included short-lived isotopes and phase transitions, but they did not consider heat of serpentinization and finite time of accretion.

Malamud and Prialnik (2013) in their recent paper compare the early evolution of Mimas and Enceladus. The aim of our paper is similar but those authors used different methods and models. Models in both papers include the same sources of heating, i.e., serpentinization, short-lived and long-lived isotopes, and gravitational energy. The main difference of their models comparing to ours is different rheology of the satellites' interior. Malamud and Prialnik (2013) assume matrix composed of rock. The flow of water through a porous rocky medium is the main process responsible for the mass transport. Water (and other liquids or gases) could flow throughout the matrix. Convection in porous media is possible but its properties are essentially different (especially efficiency of the heat transport); see Czechowski and Kossacki (2012). Of course, the sponge like model of Malamud and Prialnik (2013) includes neither solid state convection nor liquid state convection as the heat transport mechanism. The accretion is assumed to be instantaneous.

The results of Malamud and Prialnik (2013) confirm that if Enceladus accreted early (before decay of SLI) then it could be melted and differentiated into a rocky inner core and a thin icy crust. The melting of the interior led also to serpentinization of the interior. If Enceladus accreted after decay of SLI, then the melting (and serpentinization) in Enceladus could start only if ammonia was present in the ice. Malamud and Prialnik (2013) suggest that the present differences between Enceladus and Mimas could be a result of serpentinization of Enceladus during its early evolution, and the lack of this process in Mimas.

We investigate a few scenarios of Mimas and Enceladus evolutions that could lead to the present state of these satellites: dead Mimas and active 
Enceladus. We include heating from SLI and LLI as well as the heat of accretion and serpentinization. The accretion is not instantaneous, convection is considered also during accretion of the satellites. Moreover, two regimes of convection are included: liquid state convection (LSC) and solid state convection (SSC).

The paper is organized as follows. The heat sources and basic properties of MIS interior are described in Section 2. Section 3 treats about the model of convection and numerical model used for our calculations. The results are presented and discussed in Section 4. Conclusions are in the last section.

\section{HEAT SOURCES AND PROPERTIES OF MIS}

\subsection{Heat sources}

We consider the following heat sources: decay of short-lived radioactive isotopes (i.e., ${ }^{26} \mathrm{Al},{ }^{60} \mathrm{Fe},{ }^{53} \mathrm{Mn}$ ), decay of long-lived radioactive isotopes (i.e., ${ }^{40} \mathrm{~K},{ }^{232} \mathrm{Th},{ }^{238} \mathrm{U},{ }^{235} \mathrm{U}$ ), heat of serpentinization, and the heat of accretion. The latent heat of freezing/melting of ice component is also included. The tidal heating is not included because we consider only the period before the tidal heating became important. The gravitational energy released during differentiation and/or contraction is negligible for Enceladus (e.g., Malamud and Prialnik 2013).

The initial radiogenic heat rates generated by a given isotope just after the formation of CAI are given in Table 1. For any given time $t$ the heat rate is

$$
Q(t)=f_{m} \sum_{i=1}^{7} Q_{0 i} \exp \left(-C_{i} t\right),
$$

Table 1

Data concerning radioactive isotopes considered in the paper*

\begin{tabular}{|r|r|c|c|c|c|c|}
\hline Isotope & $\begin{array}{c}\text { Half life } \\
t_{1 / 2} \\
{[\mathrm{My}]}\end{array}$ & $\begin{array}{c}\text { Initial rate of } \\
\text { heating per } \\
\text { unit mass of } \\
\text { element } \\
{\left[\mathrm{W} \mathrm{kg}^{-1}\right]}\end{array}$ & $\begin{array}{c}\text { Isotopic } \\
\text { concentration } \\
\text { ppb }\left[10^{-9}\right]\end{array}$ & $\begin{array}{c}\text { Half life } \\
t_{1 / 2} \\
{[\mathrm{~s}]}\end{array}$ & $\begin{array}{c}\text { Decay } \\
\text { constant } \\
C_{-} \\
{\left[\mathrm{s}^{-1}\right]}\end{array}$ & $\begin{array}{c}\text { Initial rate of } \\
\text { heating per unit } \\
\text { mass of silicate } \\
Q_{0 i} \\
{\left[\mathrm{~W} \mathrm{~kg}{ }^{-1}\right]}\end{array}$ \\
\hline${ }^{238} \mathrm{U}$ & 4468 & $9.47 \mathrm{E}-05$ & 26.2 & $1.409 \mathrm{E}+17$ & $4.916 \mathrm{E}-18$ & $2.48 \mathrm{E}-12$ \\
${ }^{235} \mathrm{U}$ & 703.81 & $5.69 \mathrm{E}-04$ & 8.2 & $2.221 \mathrm{E}+16$ & $3.120 \mathrm{E}-17$ & $4.66 \mathrm{E}-12$ \\
${ }^{232} \mathrm{Th}$ & 14030 & $2.64 \mathrm{E}-05$ & 53.8 & $4.427 \mathrm{E}+17$ & $1.565 \mathrm{E}-18$ & $1.42 \mathrm{E}-12$ \\
${ }^{40} \mathrm{~K}$ & 1277 & $2.92 \mathrm{E}-05$ & 1104 & $4.029 \mathrm{E}+16$ & $1.720 \mathrm{E}-17$ & $3.22 \mathrm{E}-11$ \\
${ }^{26} \mathrm{AL}$ & 0.716 & $3.41 \mathrm{E}-01$ & 600 & $2.259 \mathrm{E}+13$ & $3.067 \mathrm{E}-14$ & $2.05 \mathrm{E}-07$ \\
${ }^{60} \mathrm{Fe}$ & 1.5 & $7.10 \mathrm{E}-02$ & 200 & $4.733 \mathrm{E}+13$ & $1.464 \mathrm{E}-14$ & $1.42 \mathrm{E}-08$ \\
${ }^{53} \mathrm{Mn}$ & 3.7 & $2.70 \mathrm{E}-02$ & 25.7 & $1.167 \mathrm{E}+14$ & $5.936 \mathrm{E}-15$ & $6.94 \mathrm{E}-10$ \\
\hline
\end{tabular}

*)after Robuchon et al. (2010) and Czechowski (2012) 


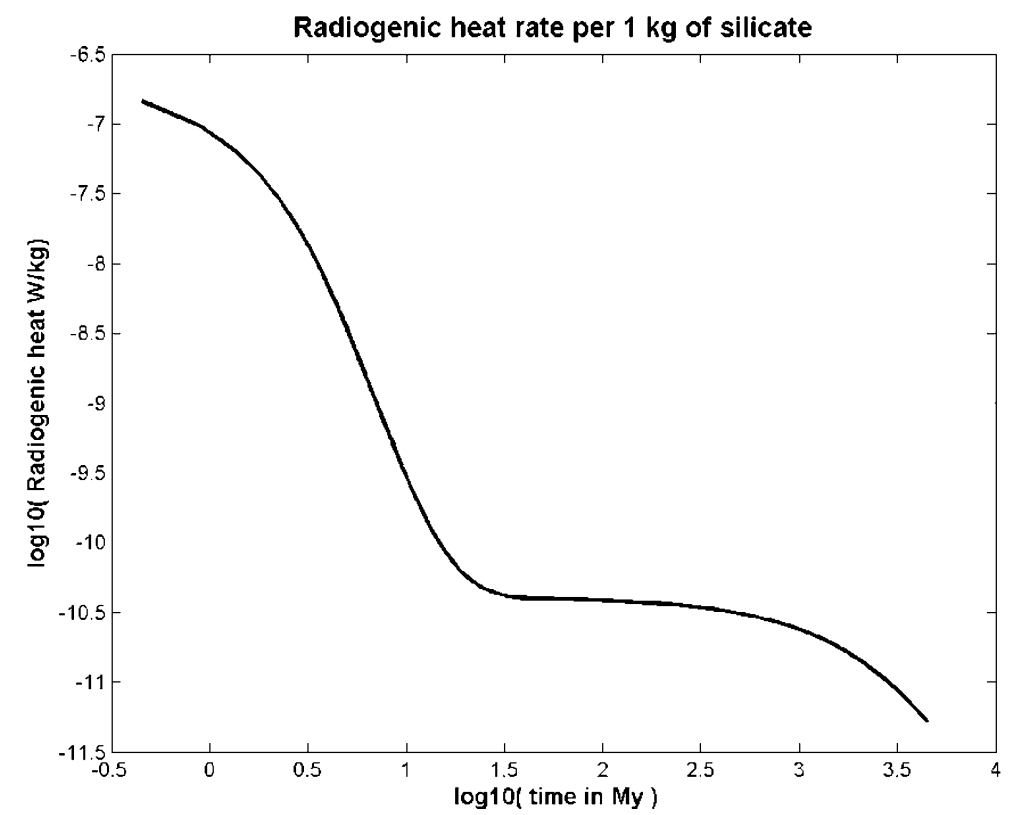

Fig. 1. Total radiogenic heat rate (in $\mathrm{W}$ per $1 \mathrm{~kg}$ of silicates of chondritic composition) versus time (from the origin of CAI). See Table 1 for isotopic composition.

where $f_{\mathrm{m}}$ is the silicate mass fraction in the satellite and time $t$ is measured from the formation of CAI (Fig. 1).

The heat of accretion results from kinetic energy of the falling matter. The temperature increase resulting from this process could be calculated from the formula (e.g., Multhaup and Spohn 2007):

$$
T_{a c}=\left(\frac{4}{3} c_{p}\right) \xi \pi G \rho r^{2}(t)\left(1+\frac{1}{2 e_{s}}\right)+T_{\text {nebula }},
$$

where $G=6.67 \times 10^{-11} \mathrm{~N} \mathrm{~m}^{2} \mathrm{~kg}^{-2}$ is the gravity constant, $\rho$ is the density, $r(t)$ is the current radius of accreting body, and $c_{p}$ is the specific heat. We adopt also here $\xi=0.4$, and the Safronov number $e_{S}=2$ after Multhaup and Spohn (2007). The surface temperature of both satellites and the nebula temperature are assumed to be the same, namely $T_{s}=T_{\text {nebula }}=75 \mathrm{~K}$.

After Abramov and Mojzsis (2011) we consider the following reaction of serpentinization:

$\mathrm{Mg}_{2} \mathrm{SiO}_{4}$ (forsterite) $+\mathrm{MgSiO}_{3}$ (enstatite) $+2 \mathrm{H}_{2} \mathrm{O} \rightarrow \mathrm{Mg}_{3} \mathrm{Si}_{2} \mathrm{O}_{5}(\mathrm{OH})_{4}$ (antigorite) 
This reaction releases $241000 \mathrm{~J} / \mathrm{kg}$ of serpentine produced. The density of serpentine is $2470 \mathrm{~kg} \mathrm{~m}^{-3}$. Moreover, we assume that silicates contain also $20 \%$ of nonreactive rock of $3630 \mathrm{~kg} \mathrm{~m}^{-3}$ density (Abramov and Mojzsis 2011). Malamud and Prialnik (2013) considered also reaction for olivine containing $80 \% \mathrm{Mg}$ and $20 \% \mathrm{Fe}$ :

$$
\mathrm{Fe}_{0.4} \mathrm{Mg}_{1.6} \mathrm{SiO}_{4}+1.23 \mathrm{H}_{2} \mathrm{O} \rightarrow 0.5 \mathrm{Mg}_{3} \mathrm{SiO}_{5}(\mathrm{OH})_{4}+0.1 \mathrm{Mg}(\mathrm{OH})_{2}+0.1 .33 \mathrm{~F}_{3} \mathrm{O}_{4}+0.133 \mathrm{H}_{2}
$$

The energy release is similar to Eq. 3. Note that we are interested mainly in energy release, and other details of reactions are not especially important.

\subsection{Rheology}

The volume percentage of rocky component in Enceladus is approximately $40 \%$. It means that rheological properties of its undifferentiated interior are determined by the icy component; according to the Roscoe's theory (e.g., Roscoe 1952, Schubert et al. 1986, Czechowski 2006a), the silicate particles form interconnecting matrix only if silicates concentration is at least $60 \%$ by volume. The rheology of icy satellites is typical for inner planets. The uppermost layer ("lithosphere") is probably elastic for small deformation and brittle for large deformations. The medium below the lithosphere is also solid but for very slow geologic processes it behaves like a viscous fluid with effective viscosity $\eta(T)$.

There are no observational data concerning the viscosity of the solid interior of MIS. Therefore we should investigate the problem for some range of viscosity. We started from the formula:

$$
\eta(T)=\eta_{x} \sigma^{(1-i)} \exp (E / R T),
$$

where $\eta_{x}$ is a constant, $\sigma$ is the second invariant of the deviatoric stress tensor, $i$ is the power law index ( $i=1$ corresponds to a Newtonian fluid), $E$ is the activation energy of the dominant mechanism of deformation, and $R=$ $8.314 \mathrm{~J} \mathrm{~K}^{-1}$ mole $^{-1}$ is the universal gas constant (McKinnon 1998, Goldsby and Kohlstedt 1997, Durham et al. 1998, Forni et al. 1991). Parameters $\eta_{x}$, $E$, and $i$ depend on many factors; e.g., size of ice crystals, content of gases, size of mineral grains, etc., most of them being essentially unknown. Durham et al. (1998) give two extreme values of $E: 43 \mathrm{~kJ} \mathrm{~mole}^{-1}$ (for water ice I below $195 \mathrm{~K}$ ) and $107.5 \mathrm{~kJ} \mathrm{~mole}^{-1}$ (for $\mathrm{NH}_{3} 2 \mathrm{H}_{2} \mathrm{O}$ ). These values correspond to $E / R$ of 5172 and $12930 \mathrm{~K}$, respectively. Rheology of both materials is highly nonlinear. Fortunately, non-Newtonian flow could be simulated by Newtonian flow with lower $E$ (Christensen 1984, Dumoulin et al. 1999); therefore we assume that $i=1$ (i.e., Newtonian flow) but we use $E=$ 
$50 \mathrm{~kJ}$ mole ${ }^{-1}$. More complicated rheology is not necessary for our calculation (see also: Davaille and Jaupart 1993, Grasset and Parmentier 1998).

The viscosity $\eta_{m}$ for the melting temperature $T_{m}$, and the activation energy $E$ are used as parameters of our model; $\eta_{m}$ is equal to

$$
\eta_{m}=\eta\left(T_{m}\right)=\eta_{x} \exp \left(E / R T_{m}\right) .
$$

\subsection{Two regimes of convection}

Two types of large scale motion in the MIS interior are considered in the present paper: solid state thermal convection (SSC), and thermal convection in liquid (LSC - liquid state convection) - Fig. 2. The SSC could develop in the upper solid layer (mantle), while LSC operates in the molten part.

The SSC is a very slow convection observed in media that are solid (from microscopic point of view) but behave like liquid in specific conditions. A few mechanisms of creep are possible, e.g., Herring-Nabarro creep and/or Cobble creep. The very large value of the Prandtl number $\mathrm{Pr}$ is characteristic for SSC (e.g., Czechowski 1993, p. 176):



Fig. 2. Scheme of structure of modeled satellites. Two possible regions are considered: molten core and solid mantle. In the molten core (if it exists at all) adiabatic gradient is maintained by liquid state convection (LSC) for most of the considered time. In the solid mantle, solid state convection (SSC) is possible if Rayleigh number $R a$ is high enough. 


$$
\operatorname{Pr}=\eta / \rho \kappa,
$$

where $\eta[\mathrm{Pas}]$ denotes the apparent viscosity, $\kappa\left[\mathrm{m}^{2} \mathrm{~s}^{-1}\right]$ is the thermal diffusivity, and $\rho\left[\mathrm{kg} \mathrm{m}^{-3}\right]$ is the density. For solid state convection, $\operatorname{Pr}=\mathrm{O}\left(10^{18}\right)$. It means that inertial terms in the equation of motion (e.g., Coriolis force) could be neglected.

Because mantle in MIS is heated mainly from below (from melted core) we use the following definition of the Rayleigh number $R a$ (e.g., Czechowski 1993, p. 175; Schubert et al. 2001, p. 270):

$$
R a=r g_{r} a c_{p} \Delta T d^{3} /\left(\kappa h_{r}\right),
$$

where $g$ is the average gravity in the considered layer $\left[\mathrm{m} \mathrm{s}^{-2}\right], \alpha$ is the coefficient of thermal volume expansion $\left[\mathrm{K}^{-1}\right], c_{p}$ is the average specific heat $\left[\mathrm{J} \mathrm{kg}^{-1} \mathrm{~K}^{-1}\right], \kappa=k /\left(\rho c_{p}\right)$ is the average value of coefficient of temperature diffusion, $k$ is the average thermal conductivity $\left[\mathrm{W} \mathrm{m}^{-1} \mathrm{~K}^{-1}\right], \Delta T$ is the temperature difference $[\mathrm{K}]$ across the considered layer, and $\eta(T)$ is the effective (temperature dependent) viscosity [Pas]. Viscosity $\eta$ is evaluated for the average temperature $T_{\text {av }}$ in the considered region. Other non-constant parameters are also averaged over the considered region.

In the above equation, $\Delta T=T_{\mathrm{m}}-T_{s}$ where $T_{s}$ is the surface temperature and $T_{\mathrm{m}}$ is the temperature of the molten region (or the temperature in the center of the satellite). Convection starts if the Rayleigh number exceeds its critical value $R a_{\mathrm{cr}}$. This value depends on situation, but usually $R a_{\mathrm{cr}}=\mathrm{O}(1000)$, (see, e.g., Czechowski 1993, p. 180 for more detailed discussion), so we choose $R a_{\mathrm{cr}}=1000$.

LSC could take place in a molten region only. The Prandtl number is $\operatorname{Pr}=\mathrm{O}(1)$. It means that inertial forces like the Coriolis force could be important for this motion (e.g., Czechowski 2012). It is a dominant form of heat transfer in the molten core. Our preliminary calculations indicate that differentiation occurs when LSC is coming to the end. Therefore, for most of time LSC convection is heated from inside (the grains with radioactive isotopes are suspended in liquid), so we use $R a_{\text {in }}$ defined as follows:

$$
R a_{\mathrm{in}}=\alpha_{m} \rho_{m}^{2} g h^{5} Q(t) /\left(k_{m} \kappa_{m} \eta_{m}\right),
$$

where subscript $m$ denotes that given parameter is calculated for molten region of the satellite. Note that liquid water contains suspended solid, so these values are not the same as for water. The $\kappa_{m}=k_{m} /\left(\rho_{m} c_{p m}\right)$ is the average value of coefficient of temperature diffusion, and $k_{m}$ is the average thermal conductivity $\left[\mathrm{W} \mathrm{m}^{-1} \mathrm{~K}^{-1}\right.$. 


\section{NUMERICAL MODEL}

\subsection{Basic equations of the model}

In our calculations we use numerical model developed by Czechowski (2012). Its short description only is given here. For details see Czechowski $(2012,2014)$. The model is based on parameterized theory of convection combined with 1-dimensional equation of the heat transfer in spherical coordinates

$$
\rho c_{p} \frac{\partial T(r, t)}{\partial t}=\operatorname{div}(k(r, T) \operatorname{grad} T(r, t))+Q(r, T),
$$

where $r$ is the radial distance (spherical coordinate), $\rho$ is the density $\left[\mathrm{kg} \mathrm{m}^{-3}\right]$, $c_{p}\left[\mathrm{~J} \mathrm{~kg}^{1} \mathrm{~K}^{-1}\right]$ is the specific heat, $Q\left[\mathrm{~W} \mathrm{~kg}^{-1}\right]$ is the heating rate, and $k$ $\left[\mathrm{W} \mathrm{m}^{-1} \mathrm{~K}^{-1}\right]$ is the thermal conductivity. Note that $Q(r, t)$ includes sources and sinks of the heat. The heat of accretion is included as initial temperature of the accreted layer. Note that Eq. 9 is solved in time dependent region $[0, R(t)]$. During accretion the radius $R(t)$ increases in time according to the formula: $R(t)=a t$ for $t_{\mathrm{ini}}<t<t_{\mathrm{ac}}$, and $R(t)=R_{\mathrm{sat}}$ for $t>t_{\mathrm{ac}}$, i.e., after the accretion (see, e.g., Merk et al. 2002), where $t_{\text {ini }}$ denotes the beginning of accretion and $t_{\mathrm{ac}}$ denotes the duration of this process.

If the Rayleigh number in the considered layer exceeds its critical value $R a_{\text {cr }}$ then convection starts. It leads to effective heat transfer. The full description of convection is given by a velocity field and temperature distribution. However, we are interested in convection as a process of heat transport only. For SSC heat transport can be described by dimensionless Nusselt number $N u$. We use the following definition of $N u$ (e.g., Czechowski 1993, p. 185):

$$
N u=(\text { True total surface heat flow }) /(\text { Total heat flow without convection }) \text {. }
$$

The heat transport by SSC is modeled simply by multiplying the coefficient of the heat conduction in the considered layer, i.e.:

$$
k_{\text {conv }}=N u k \text {. }
$$

This approach is used successfully in parameterized theory of convection for SSC in planets and satellites (e.g., Sharpe and Peltier 1978, Peltier and Jarvis 1982, Czechowski 2006b).

The method needs some formula for $N u$. We tried here a few different sets of formulas. Our preliminary results indicate that SSC in a relatively small satellite is not as dominant heat transfer mode as for large MIS like Rhea. Therefore, for our purposes the simplest approach seems to be better suited. After Turcotte and Schubert (2002, p. 273) we use

$$
N u=1.04\left(R a / R a_{\text {crit }}\right)^{1 / 3} .
$$


Parameterization of LSC is even simpler. $R a$ in molten region is very high (usually higher than $10^{16}$ ). The LSC could be very intensive, resulting in almost adiabatic temperature gradient given by

$$
\frac{d T}{d r}=\frac{g \alpha_{m} T}{c_{p m}}
$$

where $\alpha_{m}$ and $c_{p m}$ are thermal expansion coefficient and specific heat in the molten region, $g$ is the local gravity. In Enceladus and Mimas the adiabatic gradient is low and therefore the LSC region is almost isothermal.

\subsection{Gravitational differentiation and LSC}

The melting of ice allows for differentiation of the satellite. Then silicate grains sink in the molten core. However, we have also the opposite process: mixing by LSC. The interaction of these processes was considered by Czechowski (2014). His calculations indicate that convection is strong enough for mixing grains of $\sim 1 \mathrm{~mm}$ size and consequently not allowing for core formation (of course, with the exception of largest grains) for more than $100 \mathrm{Myr}$, i.e., even after the decay of most of the SLI. Convection eventually slows down and stops when the Rayleigh number drops below its critical value. Then the bulk of the core is formed. Note also that the convection and sinking process itself are strong factors separating grains according to their size, so one can expect the size of grains (and pores) to increase with depth. The relatively cold core (temperature is close to the melting point of water) of loosely packed grains with water between them is a result of this differentiation. At that time, there is no mechanism of removing the water. The chemical processing of solidification of the grains is possible but due to low temperature is rather slow. However, the concentration of silicates with radioactive elements allows for increasing the temperature in time.

The rheology of the core could be a crucial factor for tidal heating. Because the grains are mostly not glued, tidal deformations and efficient dissipation of energy are possible. In Enceladus the later evolution of core could be a result of strong tidal heating that could lead to removing water from the pores and even to dehydratation (if temperature exceed $800 \mathrm{~K}$ ). That processes could lead eventually to rigid core of large density and of relatively small size (Schubert et al. 2007). However, another option is also possible: the core still contains large amount of water and serpetinized silicates of low density (e.g., Malamud and Prialnik 2013). Calculation of Czechowski (2014) and interpretation of gravity data from Cassini (Taubner et al. 2014) indicate rather low density core of Enceladus. It means that the second option is more probable. The hypothetical core of Mimas is probably unchanged since its origin. 


\section{RESULTS AND DISCUSSION}

\subsection{Example of evolution}

Figure 3 presents an example of thermal evolution of Mimas for the following values of parameters: $\eta_{\mathrm{m}}$ is $10^{14} \mathrm{Pas}, \quad c_{\text {hyd }}=240000 \mathrm{~J} \mathrm{~kg}^{-1}, \quad E=$ $5 \times 10^{4} \mathrm{~J} \mathrm{~mole}^{-1}, t_{\mathrm{ac}}=0.1 \mathrm{Myr}, k_{\mathrm{sil}}=4.2 \mathrm{~W} \mathrm{~m}^{-1} \mathrm{~K}^{-1}, t_{\text {ini }}=1.8 \mathrm{Myr}$. The vertical axis gives distance from the center of satellite $\left(r / R_{\text {sat }}\right)$. The horizontal axis gives $\log _{10}$ (time in Myr). Note that initially the isotherms are going up following increasing radius of the accreting body. For the first several millions of years the temperature increases fast because of high concentration of radioactive elements (SLI). The maximum is reached in a few millions of years and subsequently isotherms corresponding to high temperature (let's say $260 \mathrm{~K}$ ) move down.

In the presented case there was no melting. However, for lower $t_{\text {ini, }}$, or for Enceladus, the melting is possible. Contrary to expectation, the molten region does not follow the isotherms. The matter reaches the melting temperature in a few Myr but the melting occurs after the next 2-3 Myr. The melting triggers serpentinization that supplies additional thermal energy. The radius of molten region reaches its maximum value only after $\sim 100$ Myr. It is also the time of stopping LSC and forming the core.



$\log 10(t$ in My)

Fig. 3. Thermal evolution of Mimas for the following values of parameters: $\eta_{\mathrm{m}}$ of $10^{14} \mathrm{~Pa} \mathrm{~s}, c_{\text {hyd }}=240000 \mathrm{~J} \mathrm{~kg}^{-1}, E=5 \times 10^{4} \mathrm{~J} \mathrm{~mole}^{-1}, t_{\mathrm{ac}}=0.1 \mathrm{Myr}, k_{\mathrm{s}} \mathrm{i}=4.2 \mathrm{~W} \mathrm{~m}^{-1} \mathrm{~K}^{-1}$, $t_{\text {ini }}=1.8 \mathrm{Myr}$. Vertical axis gives distance from the center of the satellite $\left(r / R_{\mathrm{sat}}\right)$. Horizontal axis gives $\log _{10}$ (time in Myr). Note that initially the isotherms are going up following increasing radius of the accreting body. For the first Myr's the temperature increases fast because of high concentration of the radioactive elements. The maximum is reached in a few millions of years and subsequently isotherms corresponding to high temperature (say $220 \mathrm{~K}$ ) move down. 


\subsection{Role of different parameters in evolution}

We performed calculations for Mimas and Enceladus for different values of the parameters: $t_{\text {ini }}$ (time of starting accretion), $t_{\mathrm{ac}}$ (duration of accretion), $\eta_{\mathrm{m}}$ (viscosity at melting temperature), $k_{\text {sil }}$ (thermal conductivity of the silicate grains), and $E$ (activation energy). The comparisons in Fig. 4 are done for
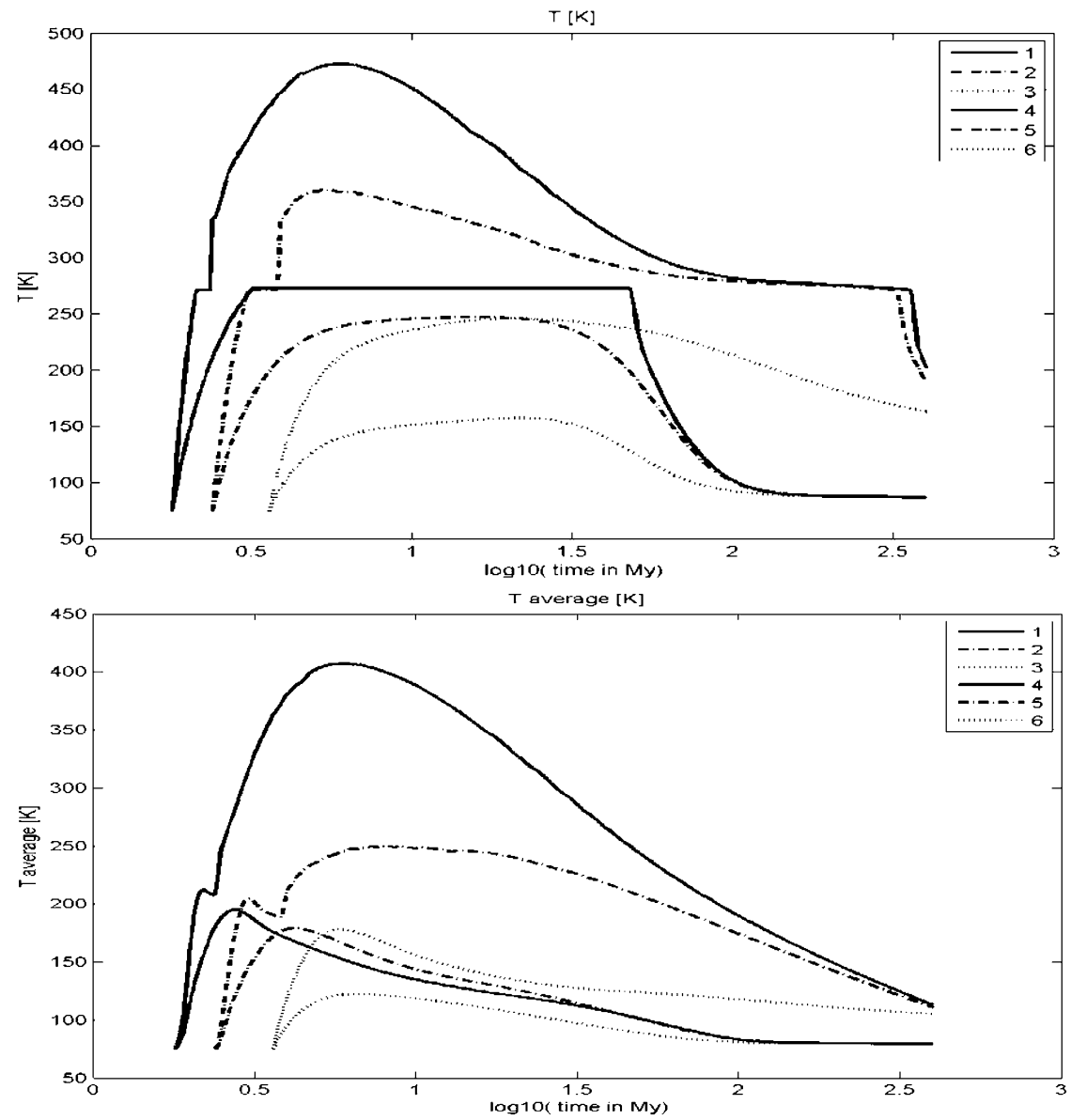

Fig. 4. Comparison of temperature in the center $T_{\max }$ (upper panel) and average temperature $T_{\text {ave }}$ (lower panel) for Enceladus and Mimas. Parameters: viscosity $\eta_{\mathrm{m}}$ of $10^{14} \mathrm{Pas}, \quad c_{\mathrm{hyd}}=240000 \mathrm{~J} \mathrm{~kg}^{-1}, \quad E=5 \times 10^{4} \mathrm{~J} \mathrm{~mole}^{-1}, \quad t_{\mathrm{ac}}=0.1 \mathrm{Myr}, \quad k_{\mathrm{sil}}=$ $4.2 \mathrm{~W} \mathrm{~m}^{-1} \mathrm{~K}^{-1}, t_{\text {ini }}=1.8,2.4,3.6 \mathrm{Myr}$ for lines 1 (and 4), 2 (and 5), 3 (and 6), respectively. Initial temperatures of both satellites are the same; note common point at $t=t_{\text {ini }}$ (leftmost). All lines for Mimas are below the corresponding lines for Enceladus indicating lower temperature in smaller Mimas. Note also that all lines for Mimas for large time converge for substantially lower temperature than the lines for Enceladus. 
the globally average temperature $T_{\mathrm{av}}$ (weighted average) and maximum temperature $T_{\max }$ (i.e., temperature in the center of a given body). The following values of parameters are chosen: viscosity $\eta_{\mathrm{m}}$ of $10^{14} \mathrm{Pas}, c_{\text {hyd }}=$ $240000 \mathrm{~J} \mathrm{~kg}^{-1}, E=5 \times 10^{4} \mathrm{~J}_{\mathrm{mole}}^{-1}, t_{\mathrm{ac}}=0.1 \mathrm{Myr}, k_{\mathrm{sil}}=4.2 \mathrm{~W} \mathrm{~m}^{-1} \mathrm{~K}^{-1}, t_{\text {ini }}=$ 1.8, 2.4, 3.6 Myr for lines 1 (and 4), 2 (and 5), 3 (and 6), respectively. Initial temperatures of both satellites are the same (note common point at $t=t_{\text {ini }}$ (leftmost)). All lines for Mimas are below the corresponding lines for Enceladus, indicating lower temperature in smaller Mimas. Note also that all lines for Mimas for large time $t$ converge to a substantially lower level than the lines for Enceladus.

Figures 5-10 present the role of other parameters. For Figure 5, the thermal conductivity $k_{\mathrm{sil}}=2.5 \mathrm{~W} \mathrm{~m}^{-1} \mathrm{~K}^{-1}$. Figures 6 and 7 give evolution of $T_{\text {ave }}$ for Enceladus and Mimas for different values of the parameters of viscosity: $\eta_{\mathrm{m}}$ is $10^{16} \mathrm{Pas}$ and $E=3 \times 10^{4} \mathrm{~J} \mathrm{~mole}^{-1}$. Figure 8 presents $T_{\text {ave }}$ for longer duration of accretion, $t_{\mathrm{ac}}=1 \mathrm{Myr}$. The energy of serpentinization is negligible for models presented in Fig. 9. Figure 10 shows evolution of the satellite with $10 \%$ of ammonia in volatiles.

Note that all the parameters discussed are important for thermal evolution. However, differences of the results are not dramatic.

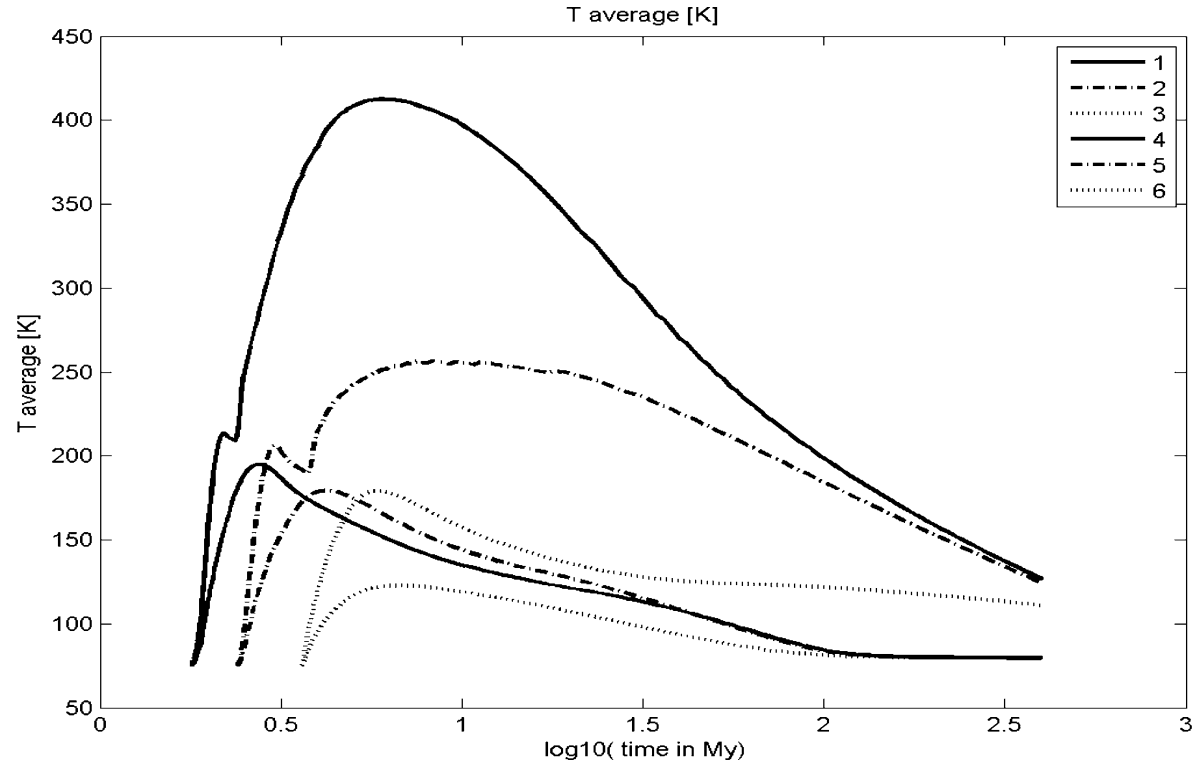

Fig. 5. Comparison of average temperature $T_{\text {ave }}$ for Enceladus and Mimas. Thermal conductivity of silicates is $k_{\text {sil }}=2.5 \mathrm{~W} \mathrm{~m}^{-1} \mathrm{~K}^{-1}$. The rest as for Fig. 4 . 


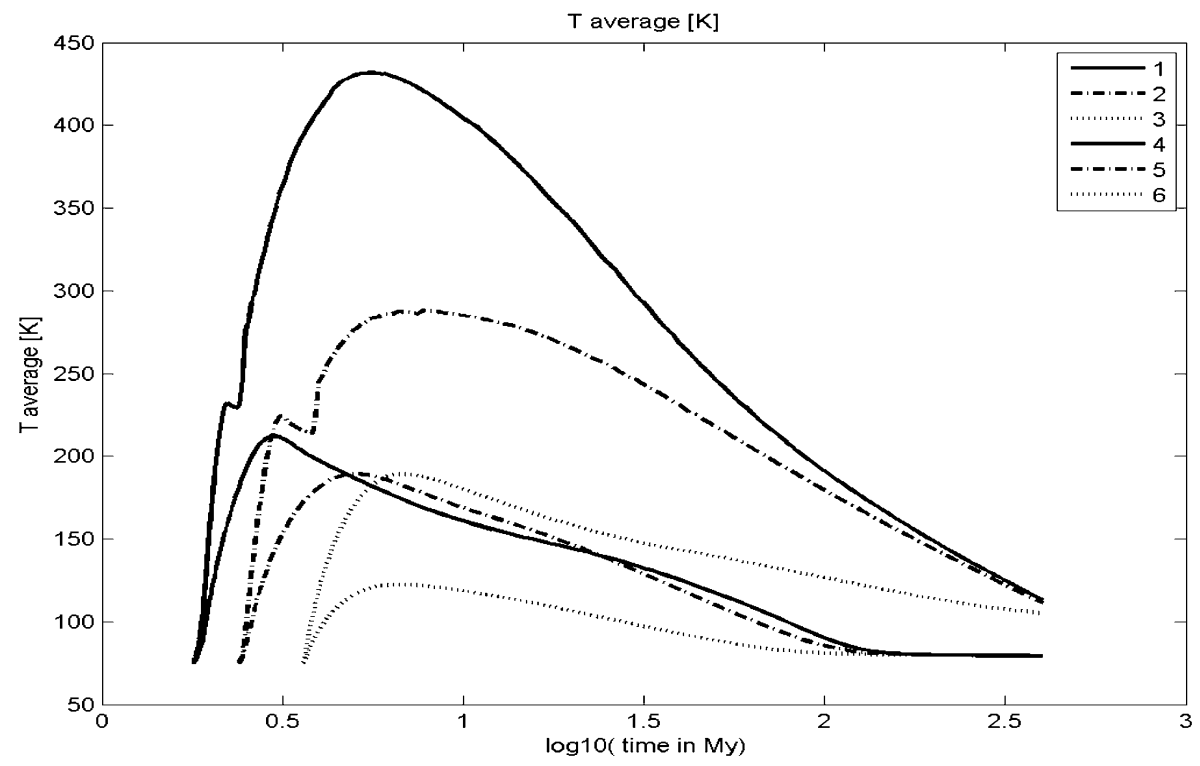

Fig. 6. Comparison of average temperature $T_{\text {ave }}$ for Enceladus and Mimas. Viscosity at melting temperature $\eta_{\mathrm{m}}$ is $10^{16} \mathrm{~Pa}$. The rest as for Fig. 4.

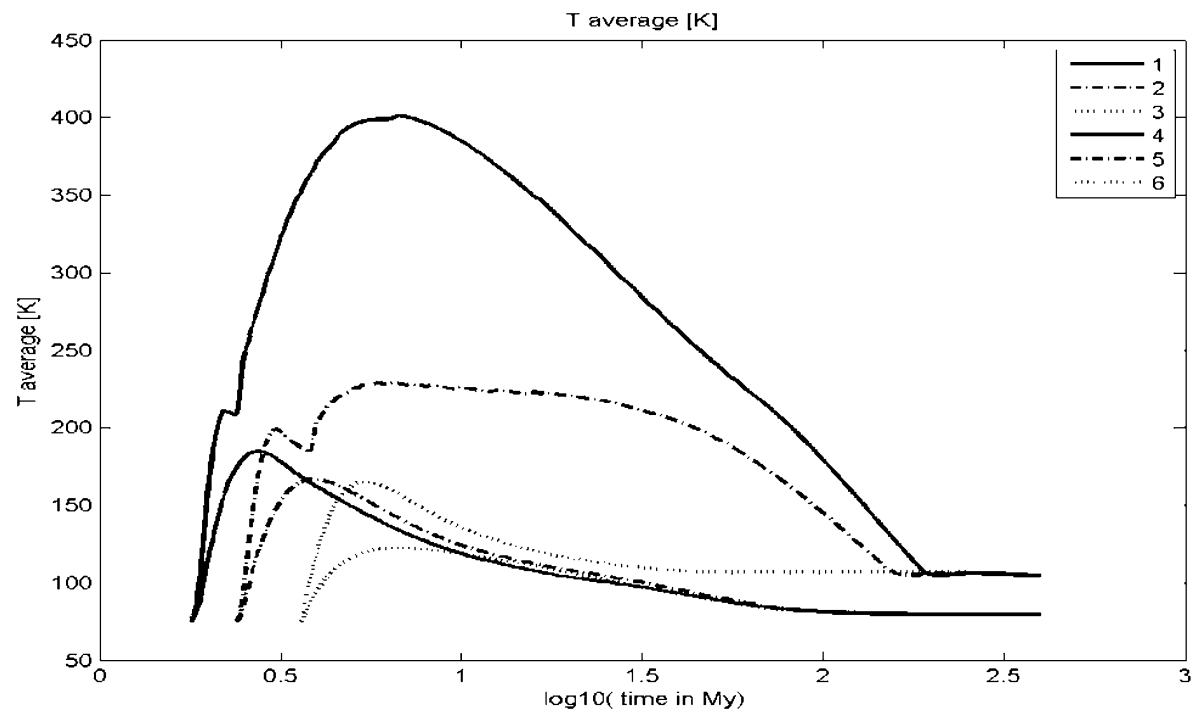

Fig. 7. Comparison of average temperature $T_{\text {ave }}$ for Enceladus and Mimas. Activation energy is $E=3 \times 10^{4} \mathrm{~J}_{\text {mole }}{ }^{-1}$. The rest as for Fig. 4 . 


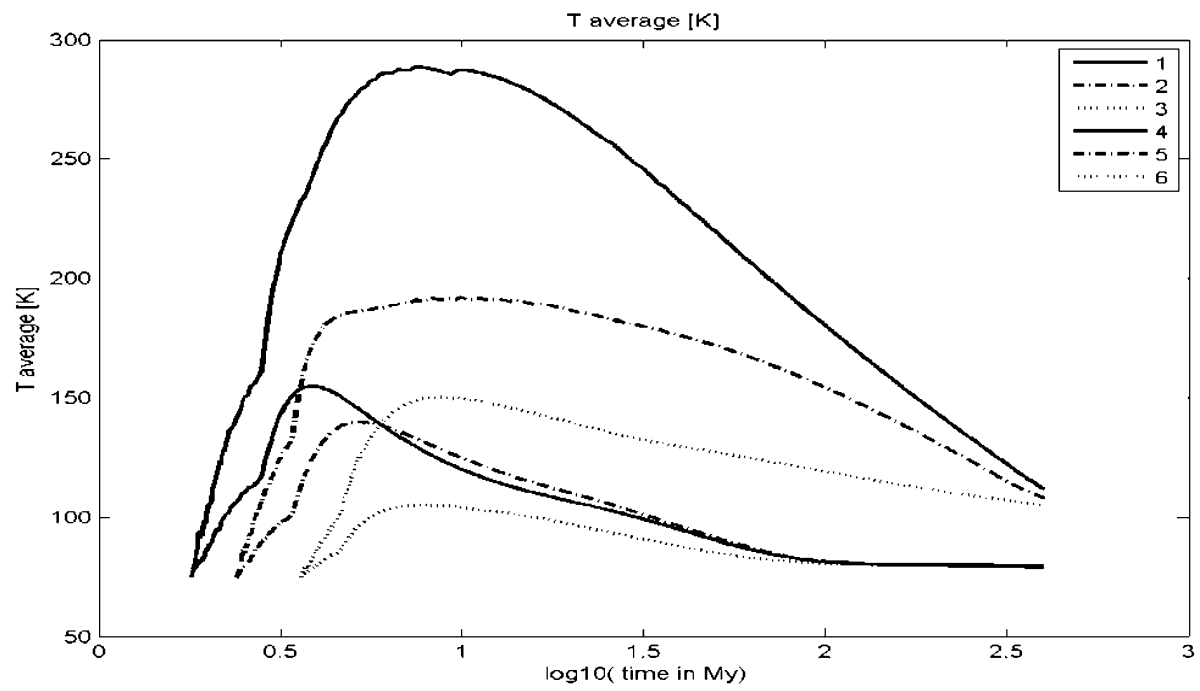

Fig. 8. Comparison of average temperature $T_{\text {ave }}$ for Enceladus and Mimas. Duration of accretion is $t_{\mathrm{ac}}=1 \mathrm{Myr}$. The rest as for Fig. 4 .

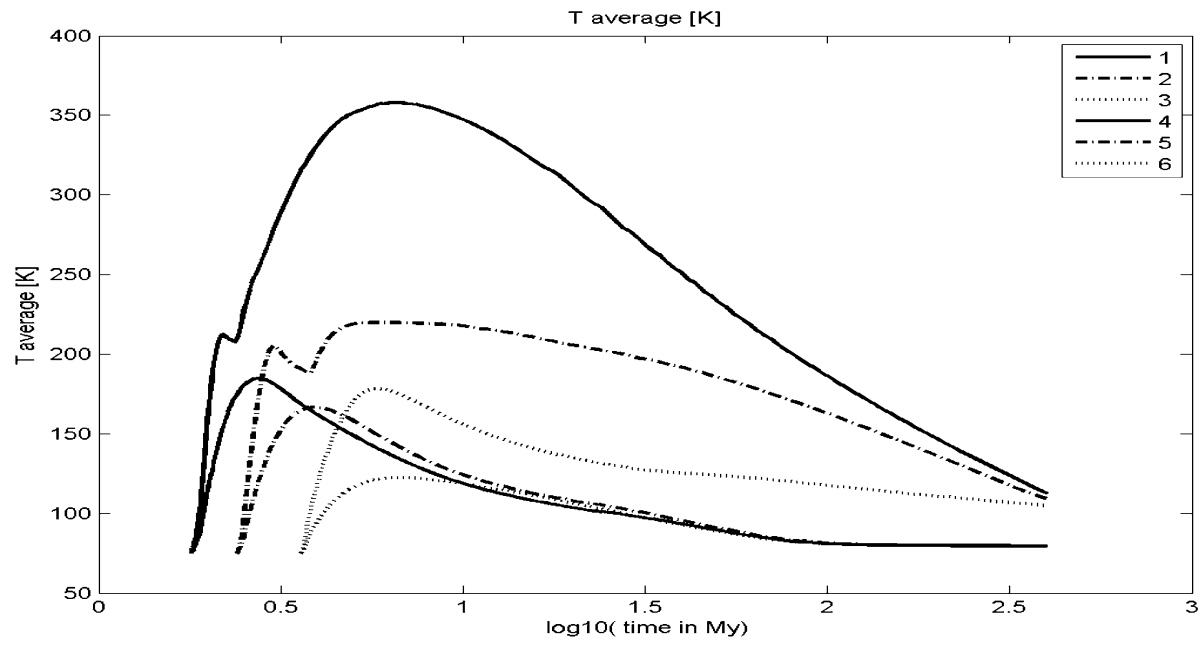

Fig. 9. Comparison of average temperature $T_{\text {ave }}$ for Enceladus and Mimas. Energy of serpentinization $c_{\text {hyd }}=0 \mathrm{~J} \mathrm{~kg}^{-1}$. The rest as for Fig. 4 . 


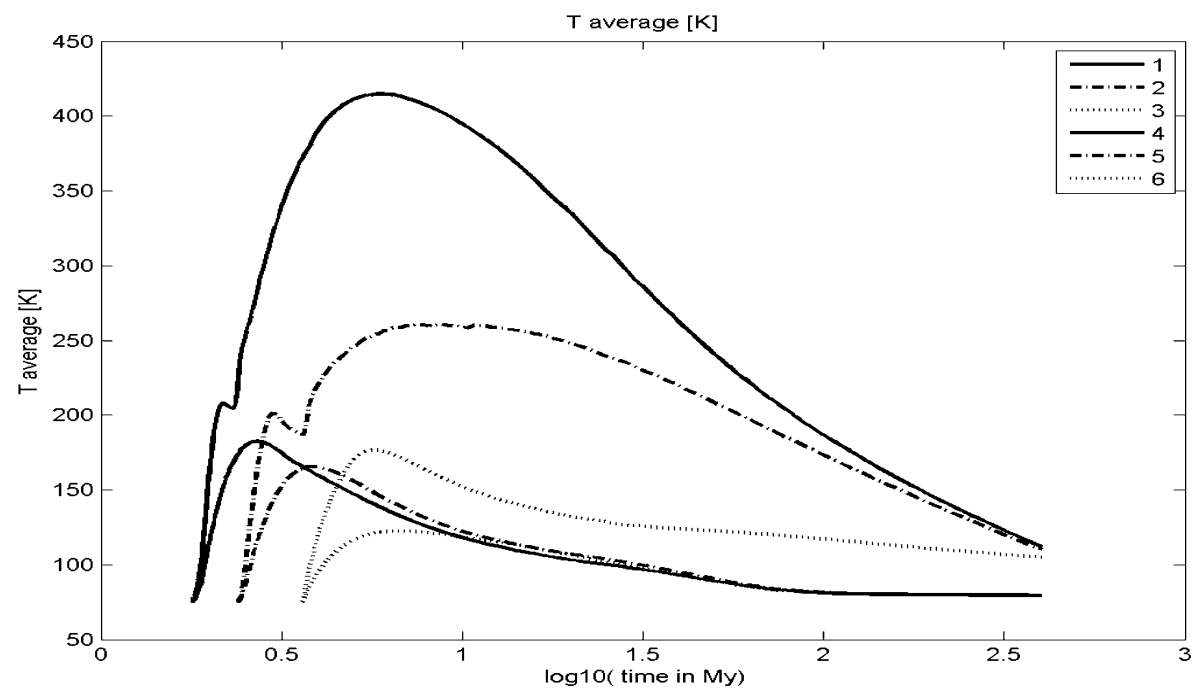

Fig. 10. Comparison of average temperature $T_{\text {ave }}$ for Enceladus and Mimas. Ammonia content is $10 \%$ of ice component. The rest as for Fig. 4.

\subsection{Mimas-Enceladus paradox}

Mimas and Enceladus are similar in size and mass and they both are in orbitorbit resonances with other MIS. However, their endogenic activities are different. Mimas is geologically dead for billions of years while Enceladus is presently active. Its activity is a result of tidal heating. For tidal heating in a satellite Poirier et al. (1983) gave the formula:

$$
q_{t \text { av }}=63 r r^{4} n^{5} e^{2} /(38 m Q),
$$

where $\rho$ is the density $\left[\mathrm{kg} \mathrm{m}^{-3}\right], r[\mathrm{~m}]$ is the satellite's radius, $n\left[\mathrm{rad} \mathrm{s}^{-1}\right]$ is the mean orbital motion, $e$ is the eccentricity of the satellite's orbit, $Q$ is the dimensionless dissipation factor, and $\mu[\mathrm{Pa}]$ is the shear modulus of the satellite's interior. Equation 14 is derived for a homogeneous body; therefore, for a non-uniform body, the effective values of $\mu$ and $Q$ should be used. The ratio of tidal heating $q_{t \text { av }}$ to total average heating $q_{\text {av }}$ is known as the dimensionless number $C=q_{t \text { av }} / q_{\text {av }}$.

Equation 14 was successfully used for Io (e.g., Peale et al. 1979). Unfortunately, the direct use of Eq. 14 to Enceladus and Mimas leads to an apparent paradox. Assuming that rheological properties of these satellites are similar (i.e., values of $\mu$ and $Q$ are the same) the products $\theta=\rho r^{4} n^{5} e^{2}$ could be treated as a measure of tidal heating. One can find that $\theta$ for active Enceladus is 31 times lower than $\theta$ for dead Mimas. This paradox indicates that a more advanced model is necessary. 
Czechowski (2006a, 2009) explained Mimas-Enceladus paradox as a result of nonlinear behavior of the system of equations describing the tidal heating, heat transfer, and rheological properties of the interior. He adopted the parameterized theory of convection for specific situation of MIS of Saturn. His model included not only temperature dependent $Q$ and $\mu$ but also possible bifurcations. The system allows for a few steady state solutions for thermal state of a given satellite. Eventually Czechowski (2006a) stated that:

- Each of the satellites has a low temperature solution that usually corresponds to low tidal heating. This state is referred to as the basic thermal state. For the smallest of MIS (i.e., Mimas or Enceladus) the temperature of this solution is very low; consequently, these satellites cannot be active in their basic states.

a Some of the satellites have also one or two additional solutions with higher temperatures and with higher rates of tidal heating than for the basic state. These additional solutions correspond to thermal states that are referred here as excited states.

The basic solution always exists and corresponds to the lowest possible temperature. The existence of excited states with high temperature does not guarantee an endogenic activity of a given satellite. It means only that the given satellite could be in one of a few thermal states; the satellite could be in its basic state even if exited states exist.

For starting efficient tidal heating it is necessary that:

1. temperature of interior is high enough for effective dissipation of tidal stresses,

2. the orbit has a significant eccentricity.

Moreover, for long lasted tidal heating it is necessary that:

3 . there occurs an orbit-orbit resonance that could keep the non-zero eccentricity.

All conditions must be fulfilled simultaneously. Presently, Mimas fulfills conditions 2 and 3. If its temperature were high enough then the tidal heating could be high and Mimas could be active. The initial temperature of the satellite could be a factor deciding upon the path of evolution.

Generally, with the exception of a short time (approximately 1-4 Myr after CAI), Mimas was always substantially colder than Enceladus. We do not know the critical temperature necessary for starting process of effective tidal heating in Mimas, but evidently the high temperature is important. We do not know when condition 3 was fulfilled, i.e., when Mimas-Tethys and Enceladus-Dione resonances were established. Note, however, that condition 1 could be satisfied in Mimas, only if it accreted early. This leads us to additional condition: $t_{\text {ini }}<1.8 \mathrm{Myr}$ for Mimas. However even if this additional condition was satisfied and Mimas was (for some time) hot enough for 
efficient tidal dissipation, the duration of this "launch window" was dramatically shorter ( $\sim 10 \mathrm{Myr})$ for Mimas than for Enceladus ( 100 Myr). Probably the Mimas-Tethys resonance was established too late to change the path of evolution of Mimas.

Taking into account early thermal history of both satellites, the present situation can hardly be treated as a paradox. The Mimas should be very "lucky" if time of resonance and time of high temperature were at the moment when Mimas was hot.

\subsection{Chronology and activity of Mimas and Enceladus}

Mimas is classified as a dead satellite according to Rothery (1992). It means that there was no substantial endogenic activity since Heavy Bombardment Epoch (HBE). Note however that the assessment of age depends on the assumed model of flux of meteorites. Using the lunar-like flux, cratered plains of Enceladus are 4.2 Gyr old and only 1.7 Gyr old, if cometary impact rate is used for calculation (Zahnle et al. 2003, Spencer et al. 2009). Saturated by craters, the surface of Mimas is probably older than 4 Gyr according to "lunar" chronology, but only $\sim 2$ Gyr if cometary impact rate is appropriate. If "short", "cometary" chronology is correct then we do not have data concerning $2.5 \mathrm{Gyr}$ of Mimas history. During that time, the satellite could be active but the older lithosphere was destroyed.

\section{CONCLUSIONS}

- We compared thermal evolution for Enceladus and Mimas for several parameters: $\eta_{m}, c_{\mathrm{hyd}}, E, t_{\mathrm{ac}}, k_{\mathrm{sil}}$, and $t_{\mathrm{ini}}$. All of these parameters are significant but the time of accretion $t_{\text {ini }}$ is the most important (see also Czechowski 2014).

- The Mimas-Enceladus paradox is probably the result of short time when Mimas was hot enough. This conclusion does not contradict the results of Malamud and Prialnik (2013) about the role of serpentinization. However, we indicate that the role of serpentinization is not critical. The paradox could exist even if serpentization of the matter had occurred before accretion.

口 If "short", "cometary" chronology is correct, then Mimas could have been active for some time.

Acknowledgements. This work was partially supported by the National Research Centre (grant 2011/01/B/ST10/06653). Computer resources of Interdisciplinary Centre for Mathematical and Computational Modeling of Warsaw University are also used in the research. We are also grateful for Dr. Anna Łosiak for her suggestions. 


\section{References}

Abramov, O., and S.J. Mojzsis (2011), Abodes for life in carbonaceous asteroids? Icarus 213, 1, 273-279, DOI: 10.1016/j.icarus.2011.03.003.

Bobojć, A., and A. Drożyner (2011), GOCE satellite orbit in the aspect of selected gravitational perturbations, Acta Geophys. 59, 2, 428-452, DOI: 10.2478/ s11600-010-0052-3.

Charnoz, S., A. Morbidelli, L. Dones, and J. Salmon (2009), Did Saturn's rings form during the Late Heavy Bombardment? Icarus 199, 2, 413-428, DOI: 10.1016/j.icarus.2008.10.019.

Christensen, U. (1984), Convection with pressure- and temperature-dependent nonNewtonian rheology, Geophys. J. Int. 77, 2, 343-384, DOI: 10.1111/j.1365246X.1984.tb01939.x.

Czechowski, L. (1993), Theoretical approach to mantle convection. In: R. Teisseyre, L. Czechowski, and J. Leliwa-Kopystyński (eds.), Dynamics of the Earth's Evolution, PWN - Polish Scientific Publ., Warszawa, Elsevier, Amsterdam, 161-271.

Czechowski, L. (2006a), Parameterized model of convection driven by tidal and radiogenic heating, $A d v$. Space Res. 38, 4, 788-793, DOI: 10.1016/j.asr.2005. 12.013 .

Czechowski, L. (2006b), Two models of parameterized convection for mediumsized icy satellites of Saturn, Acta Geophys. 54, 3, 280-302, DOI 10.2478/ s11600-006-0021-z.

Czechowski, L. (2009), Uniform parameterized theory of convection in medium sized icy satellites of Saturn, Acta Geophys. 57, 2, 548-566, DOI: 10.2478/ s11600-008-0084-0.

Czechowski, L. (2012), Thermal history and large scale differentiation of the Saturn's satellite Rhea, Acta Geophys. 60, 4, 1192-1212, DOI: 10.2478/ s11600-012-0041-9.

Czechowski, L. (2014), Some remarks on the early evolution of Enceladus, Planet. Space Sci. 104, 185-199, DOI: 10.1016/j.pss.2014.09.010.

Czechowski, L., and K.J. Kossacki (2012), Thermal convection in the porous methane-soaked regolith in Titan: finite amplitude convection, Icarus 217, 1, 130-143, DOI: 10.1016/j.icarus.2011.10.006.

Davaille, A., and C. Jaupart (1993), Transient high-Rayleigh-number thermal convection with large viscosity variations, J. Fluid Mech. 253, 141-166, DOI: 10.1017/S0022112093001740.

Dumoulin, C., M.-P. Doin, and L. Fleitout (1999), Heat transport in stagnant lid convection with temperature- and pressure-dependent Newtonian or nonNewtonian rheology, J. Geophys. Res. 104, B6, 12759-12777, DOI: 10.1029/1999JB900110. 
Durham, W.B., S.H. Kirby, and L.A. Stern (1998), Rheology of planetary ices. In: B. Schmitt, C. de Bergh, and M. Festou (eds.), Solar System Ices, Kluwer Academic Publ., Dordrecht, 63-78, DOI: 10.1007/978-94-011-5252-5_3.

Ellsworth, K., and G. Schubert (1983), Saturn's icy satellites: Thermal and structural models, Icarus 54, 3, 490-510, DOI: 10.1016/0019-1035(83)90242-7.

Eluszkiewicz, J. (1990), Compaction and internal structure of Mimas, Icarus 84, 1, 215-225, DOI: 10.1016/0019-1035(90)90167-8.

Essa, K.S. (2007), A simple formula for shape and depth determination from residual gravity anomalies, Acta Geophys. 55, 2, 182-190, DOI: 10.2478/ s11600-007-0003-9.

Forni, O., A. Coradini, and C. Federico (1991), Convection and lithospheric strength in Dione, an icy satellite of Saturn, Icarus 94, 1, 232-245, DOI: 10.1016/ 0019-1035(91)90153-K.

Goldsby, D.L., and D.L. Kohlstedt (1997), Grain boundary sliding in fine-grained Ice - I, Scripta. Mater. 37, 9, 1399-1406, DOI: 10.1016/S1359-6462(97) 00246-7.

Grasset, O., and E.M. Parmentier (1998), Thermal convection in a volumetrically heated, infinite Prandtl number fluid with strongly temperature-dependent viscosity: Implications for planetary evolution, J. Geophys. Res. 103, B8, 18171-18181, DOI: 10.1029/98JB01492.

Jaumann, R., R.N. Clark, F. Nimmo, A.R. Hendrix, B.J. Buratti, T. Denk, J.M. Moore, P.M. Schenk, S.J. Ostro, and R. Srama (2009), Icy satellites: Geological evolution and surface processes. In: M.K. Dougherty L.W. Esposito, and S.M. Krimigis (eds.), Saturn from Cassini-Huygens, Springer Science+Business Media, Dordrecht, 637-681, DOI: 10.1007/9781-4020-9217-6_20.

Kriegel, H., S. Simon, J. Müller, U. Motschmann, J. Saur, K.-H. Glassmeier, and M.K. Dougherty (2009), The plasma interaction of Enceladus: 3D hybrid simulations and comparison with Cassini MAG data, Planet. Space Sci. 57, 14-15, 2113-2122, DOI: 10.1016/j.pss.2009.09.025.

Leliwa-Kopystyński, J., and K.J. Kossacki (2000), Evolution of porosity in small icy bodies, Planet. Space Sci. 48, 7-8, 727-745, DOI: 10.1016/S0032-0633 (00)00038-6.

Malamud, U., and D. Prialnik (2013), Modeling serpentinization: Applied to the early evolution of Enceladus and Mimas, Icarus 225, 1, 763-774, DOI: 10.1016/j.icarus.2013.04.024.

Matson, D.L., J.C. Castillo-Rogez, G. Schubert, C. Sotin, and W.B. McKinnon (2009), The thermal evolution and internal structure of Saturn's mid-sized icy satellites. In: M.K. Dougherty, L.W. Esposito, and S.M. Krimigis (eds.), Saturn from Cassini-Huygens, Springer Science+Business Media, Dordrecht, 577-612, DOI: 10.1007/978-1-4020-9217-6_18. 
McKinnon, W.B. (1998), Geodynamics of icy satellites. In: B. Schmitt, C. de Bergh, and M. Festou (eds.), Solar System Ices, Kluwer Academic Publ., Dordrecht, 525-550, DOI: 10.1007/978-94-011-5252-5_22.

McKinnon, W.B., and A.C. Barr (2007), The Mimas paradox revisited plus crustal spreading on Enceladus? LPI Contrib. 1357, 91-92.

Merk, R., D. Breuer, and T. Spohn (2002), Numerical modeling of ${ }^{26}$ Al-induced radioactive melting of asteroids concerning accretion, Icarus 159, 1, 183-191, DOI: 10.1006/icar.2002.6872.

Meyer, J., and J. Wisdom (2008), Tidal evolution of Mimas, Enceladus, and Dione, Icarus 193, 1, 213-223, DOI: 10.1016/j.icarus.2007.09.008.

Multhaup, K., and T. Spohn (2007), Stagnant lid convection in the mid-sized icy satellite of Saturn, Icarus 186, 2, 420-435, DOI: 10.1016/j.icarus.2006.09. 001.

Muro, G.D., and F. Nimmo (2011), Modeling the coupled thermal and orbital evolution of Mimas, LPI Contrib. 1608, 1560.

Peale, S.J. (2003), Tidally induced volcanism, Celest. Mech. Dyn. Astr. 87, 1-2, 129155, DOI: 10.1023/A:1026187917994.

Peale, S.J., P. Cassen, and R.T. Reynolds (1979), Melting of Io by tidal dissipation, Science 203, 4383, 892-894, DOI: 10.1126/science.203.4383.892.

Peltier, W.R., and G.T. Jarvis (1982), Whole mantle convection and the thermal evolution of the Earth, Phys. Earth Planet. In. 29, 3-4, 281-304, DOI: 10.1016/ 0031-9201(82)90018-8.

Poirier, J.P., L. Boloh, and P. Chambon (1983), Tidal dissipation in small viscoelastic ice moons: The case of Enceladus, Icarus 55, 2, 218-230, DOI: 10.1016/ 0019-1035(83)90076-3.

Robuchon, G., G. Choblet, G. Tobie, O. Čadek, C. Sotin, and O. Grasset (2010), Coupling of thermal evolution and despinning of early Iapetus, Icarus 207, 2, 959-971, DOI: 10.1016/j.icarus.2009.12.002.

Roscoe, R. (1952), The viscosity of suspensions of rigid spheres, British J. Appl. Phys. 3, 8, 267-269, DOI: 10.1088/0508-3443/3/8/306.

Rothery, D.A. (1992), Satellites of the Outer Planets: Worlds in Their Own Right, Clarendon Press, Oxford, 208 pp.

Schubert, G., T. Spohn, and R.T. Reynolds (1986), Thermal histories, compositions and internal structures of the moons of the solar system. In: J.A. Burns and M.S. Matthews (eds.), Satellites, University of Arizona Press, Tucson, 224292.

Schubert, G., D.L. Turcotte, and P. Olson (2001), Mantle Convection in the Earth and Planets, Cambridge Univ. Press, Cambridge, 956 pp.

Schubert, G., J.D. Anderson, B.J. Travis, and J. Palguta (2007), Enceladus: Present internal structure and differentiation by early and long-term radiogenic heating, Icarus 188, 2, 345-355, DOI: 10.1016/j.icarus.2006.12.012. 
Sharpe, H.N., and W.R. Peltier (1978), Parameterized mantle convection and the Earth's thermal history, Geophys. Res. Lett. 5, 9, 737-740, DOI: 10.1029/ GL005i009p00737.

Spencer, J.R., A.C. Barr, L.W. Esposito, P. Helfenstein, A.P. Ingersoll, R. Jaumann, C.P. McKay, F. Nimmo, and J.H. Waite (2009), Enceladus: An active cryovolcanic satellite. In: M.K. Dougherty, L.W. Esposito, and S.M. Krimigis (eds.), Saturn from Cassini-Huygens, Springer Science+Business Media, Dordrecht, 683-724, DOI: 10.1007/978-1-4020-9217-6_21.

Taubner, R.S., J.J. Leitner, M.G. Firneis, and R. Hirzenberger (2014), Including Cassini's gravity measurements from the flybys E9, E12, E19 into interior structure models of Enceladus. In: Proc. European Planetary Science Congress, 7-12 September 2014, Cascais, Portugal, EPSC Abstracts, 2014-676.

Thomas, P.C. (2010), Sizes, shapes, and derived properties of the Saturnian satellites after the Cassini nominal mission, Icarus 208, 1, 395-401, DOI: 10.1016/ j.icarus.2010.01.025.

Turcotte, D.L., and G. Schubert (2002), Geodynamics, 2nd ed., Cambridge University Press, Cambridge, 465 pp.

Zahnle, K., P. Schenk, H. Levison, and L. Dones (2003), Cratering rates in the outer Solar System, Icarus 163, 2, 263-289, DOI: 10.1016/S0019-1035(03) 00048-4.

Received 19 November 2013

Received in revised form 7 January 2015

Accepted 19 January 2015 